\title{
Comorbidade entre depressão, ansiedade e obesidade e complicações no tratamento
}

\author{
Comorbidity between depression, anxiety and obesity and treatment complications \\ Comorbilidad entre depresión, ansiedad y obesidad y complicaciones del tratamiento
}

Recebido: 25/12/2020 | Revisado: 31/12/2020 | Aceito: 04/01/2021 | Publicado: 06/01/2021

\author{
Daniel Del Nero Casselli \\ ORCID: https://orcid.org/0000-0003-4595-9701 \\ Universidade Federal do Triângulo Mineiro, Brasil \\ E-mail: ddncasselli@gmail.com \\ Eduardo de Sousa Martins e Silva \\ ORCID: https://orcid.org/0000-0001-6257-4681 \\ Universidade Federal do Triângulo Mineiro, Brasil \\ E-mail: eduardosousa25@gmail.com \\ Gabriela Martins Figueira \\ ORCID: https://orcid.org/0000-0002-8995-1755 \\ Universidade Federal do Triângulo Mineiro, Brasil \\ E-mail: gabrielamfigueira19@gmail.com \\ Mariana Eduarda Demarchi \\ ORCID: https://orcid.org/0000-0002-9980-8709 \\ Universidade Federal do Triângulo Mineiro, Brasil \\ E-mail: marianaedemarchi@hotmail.com \\ José Carlos Souza \\ ORCID: https://orcid.org/0000-0003-4460-3770 \\ Universidade Estadual de Mato Grosso do Sul, Brasil \\ E-mail: josecarlossouza@uol.com.br
}

\begin{abstract}
Resumo
Os transtornos mentais, como o Transtorno Depressivo Maior e os Transtornos de Ansiedade, juntamente da obesidade, têm crescido, substancialmente, no último século. O aumento do consumo de alimentos ricos em gorduras e em açúcares e do sedentarismo, assim como fatores genéticos e cotidianos parecem explicar, até certo grau, a incidência de doenças metabólicas e da obesidade. Não obstante, o melhor entendimento dos fatores de risco relacionados ao aparecimento de desordens psíquicas, em certas populações, acarreta mudanças no tratamento destas patologias, favorecendo um cuidado integral e multidisciplinar. Dentro deste escopo, o presente estudo pretende analisar se há correlação entre depressão, ansiedade e obesidade, quando se trata do tratamento multidisciplinar destas patologias. Fez-se uma revisão bibliográfica integrativa usando-se artigos dos últimos 10 anos (2010-2020) das bases de dados da Literatura Latino-Americana e do Caribe em Ciências da Saúde - LILACS, PubMed, Cochrane e Biblioteca Eletrônica Científica Online - SciELO, com os descritores: "Obesidade"; "Transtornos Mentais"; "Ansiedade"; "Depressão"; "Comorbidades".A literatura apresenta discordâncias na associação entre obesidade, ansiedade e depressão. Alguns estudos demonstram que não há correlação estatística significativa entre IMC, depressão e ansiedade. Do mesmo modo, outros estudos demonstram que a correlação entre obesidade e transtornos de humor é influenciada pela utilização de psicofármacos empregados no tratamento psiquiátrico, com potencial de influenciar no ganho de peso. As relações de causalidade envolvendo depressão, ansiedade e obesidade ainda são controversas e requerem mais pesquisa.
\end{abstract}

Palavras-chave: Obesidade; Transtornos mentais; Ansiedade; Depressão; Comorbidades.

\begin{abstract}
Mental disorders such as Major Depressive Disorder and Anxiety Disorders, along with obesity, have grown substantially in the last century. The increase in the consumption of foods rich in fats and sugars and sedentary lifestyle, as well as genetic and everyday factors seem to explain, to some degree, the incidence of metabolic diseases and obesity. Nevertheless, a better understanding of risk factors related to the onset of psychic disorders in certain populations lead to changes in the treatment of these pathologies, favoring comprehensive and multidisciplinary care. Within this scope, the present study aims to analyze whether there is a correlation between depression, anxiety and obesity, when it comes to the multidisciplinary treatment of these pathologies. An integrative bibliographic review was made using articles from the last 10 years (2010-2020) from the databases of Latin American and Caribbean Literature on Health Sciences - LILACS, PubMed, Cochrane and Online Scientific Electronic Library - SciELO, with the descriptors: "Obesity"; "Mental Disorders"; "Anxiety"; "Depression"; "Comorbidities." The literature presents disagreements in the association between obesity, anxiety and depression. Some studies show that there is no statistically significant correlation between BMI, depression and anxietyon. Likewise, other studies show that the correlati between obesity and mood disorders is influenced by the use of psychotropic drugs used in psychiatric
\end{abstract}


treatment, with the potential to influence weight gain. The causal relationships involving depression, anxiety and obesity are still controversial and require more research.

Keywords: Obesity; Mental disorders; Anxiety; Depression; Comorbidities.

\begin{abstract}
Resumen
Los trastornosmentales, como eltrastornodepresivomayor y lostrastornos de ansiedad, junto conlaobesidad, hancrecidosustancialmenteenel último siglo. El aumento del consumo de alimentos ricos engrasas y azúcares y lainactividad física, así como factores genéticos y cotidianos parecen explicar, encierta medida, laincidencia de enfermedades metabólicas y obesidad. No obstante, una mejorcomprensión de losfactores de riesgo relacionados conlaaparición de trastornos psíquicos, en determinadas poblaciones, conduce a cambioseneltratamiento de estas patologías, favoreciendolaatención integral y multidisciplinar. En este ámbito, el presente estudiotiene como objetivo analizar si existe una correlación entre depresión, ansiedad y obesidad, enlo que respecta al tratamientomultidisciplinario de estas patologías. Se realizó una revisión bibliográfica integradora utilizando artículos de los últimos 10 años (2010-2020) de las bases de datos de Literatura Latinoamericana y del Caribe enCiencias de laSalud - LILACS, PubMed, Cochrane y ElectronicScientific Library Online - SciELO, conlosdescriptores: "Obesidad"; "Desordenes mentales"; "Ansiedad"; "Depresión"; "Comorbilidades". La literatura muestradesacuerdosenlaasociación entre obesidad, ansiedad y depresión. Algunosestudiosmuestran que no existe una correlaciónestadísticamente significativa entre el IMC, ladepresión y laansiedad. Asimismo, otrosestudiosmuestran que lacorrelación entre laobesidad y lostrastornosdel estado de ánimo está influenciada por el uso de psicofármacos utilizados eneltratamiento psiquiátrico, conel potencial de influir enel aumento de peso. Las relaciones causales que involucrandepresión, ansiedad y obesidadaúnson controvertidas y requieren más investigación.
\end{abstract}

Palabras clave: Obesidad; Desordenes mentales; Ansiedad; Depresión; Comorbilidades.

\title{
1. Introdução
}

Os transtornos alimentares, em geral, costumam ter como comorbidades frequentes a ansiedade e a depressão; entre estes destaca-se a obesidade (Almeida et al., 2012). Muitos pacientes obesos apresentam, inicialmente, um quadro depressivo e/ou ansioso e evolui para um aumento de peso significativo, podendo chegar à obesidade mórbida (Rocha \& Costa, 2012). O Transtorno Compulsivo Alimentar Periódico é um dos exemplos de como a ansiedade para a ingestão de alimentos, geralmente com excesso de carboidratos, são ingeridos em um curto espaço de tempo com o aumento significativo do peso corporal. Estas situações interferem no tratamento, mesmo que multidisciplinar, e no prognóstico destes quadros clínicos comórbidos (Garcia et al., 2018).

Deve-se levar em conta o atual conceito ampliado de saúde, desde a abordagem, o diagnóstico, diagnósticos diferenciais e tratamento multi e transdisciplinar da obesidade; o clínico não deve deixar de elencar as comorbidades, os fatores de risco e predisponentes deste importante problema de saúde pública, o qual tem aumentado significativamente; como também, a obesidade tem sido a causa de morte cada vez mais freqüente entre adultos jovens e crianças com a mais tenra idade (Frontzek, 2017). Por conta disso, convém que a avaliação do paciente seja individualizada, o mais abrangente e integral possível, levando-se em consideração os seus aspectos físicos, biológicos, sociais, ambientais, históricos, psicológicos, culturais e espirituais (no sentido de crenças), visando um diagnóstico o mais acurado possível, o tratamento necessário e sem complicações, para um prognóstico satisfatório (Mensorio\& Junior, 2016). Neste contexto, este estudo tem como objetivo discutir os aspectos essenciais do tratamento da obesidade, quando comórbida com a ansiedade e a depressão, relacionando a presença destas comorbidades com complicações no tratamento.

\section{Metodologia}

O presente trabalho, de natureza quantitativa, trata-se de uma revisão integrativa da literatura e tem por propósito congregar e examinar os resultados de estudos independentes acerca do tema "Comorbidade entre depressão, ansiedade e obesidade e complicações no tratamento", norteando-se pela pergunta de pesquisa estabelecida pelos autores: "Há possibilidade de correlação entre depressão, ansiedade e obesidade, quando se trata do tratamento 
farmacológico/multidisciplinar dessas patologias?”.

Neste estudo, utilizou-se o método proposto por Whittemore e Knafl's (2005) para revisões integrativas de literatura. Posto a possibilidade de inclusão de estudos com desenhos variados e a multiplicidade de propostas, aliados à falta de métodos sistematizados, Whittemore e Knafl's preconizaram cinco etapas, com a finalidade de expandir a precisão metodológica das revisões integrativas de literatura. De acordo com estes autores, precisa-se, inicialmente, reconhecer o problema principal do estudo, tal como sua finalidade. Posteriormente, devem ser definidos, de modo nítido, os procedimentos de pesquisa descritores utilizados, bases de dados, critérios de inclusão, entre outros. Depois, é necessário que a verificação da qualidade dos trabalhos englobados seja feita, mesmo que não haja padrão-ouro para as revisões integrativas. Em seguida, as informações retiradas dos trabalhos devem ser organizadas, classificadas e sumarizadas em uma conclusão unificada acerca do problema abordado na revisão. Por último, deve-se exibir as apurações e as conclusões obtidas.

Com a finalidade de nortear o estudo, preconizou-se que o público-alvo se restringiria a indivíduos com uma ou mais destas características: Índice de Massa Corporal (IMC) maior ou igual a $30 \mathrm{~kg} / \mathrm{m} 2$, acometidos por Transtorno Depressivo Maior (em tratamento), acometidos por Transtornos de Ansiedade (em tratamento). Não obstante, os resultados apresentados neste trabalho devem sugerir uma solução para a pergunta norteadora do estudo. Com base nisto, devem debater sobre a possibilidade de haver correlações entre depressão, ansiedade e obesidade durante o tratamento destas patologias.

Para a finalização do trabalho, fez-se uma busca integrativa e, em seguida, uma escolha das publicações indexadas em quatro bases de dados: Literatura Latino-Americana e do Caribe em Ciências da Saúde - LILACS, PubMed, Cochrane e Biblioteca Eletrônica Científica Online - SciELO. Os descritores utilizados foram: "Obesidade", "Transtorno Mental", "Depressão", "Ansiedade" e "Comorbidades", com operadores booleanos "e" e "ou". Adotou-se os seguintes critérios de inclusão: 1) artigos originais (estudos experimentais, ensaios clínicos randomizados, estudos caso-controle); 2) artigos publicados na língua inglesa e na língua portuguesa; 3) artigos publicados no ano de 2010 até 2020; 4) artigos que possuíam no título e/ou resumo os descritores utilizados na busca.

A pesquisa foi concluída em outubro de 2020 e, ao todo, foram obtidos 273 artigos científicos. Realizou-se uma leitura dinâmica dos resumos de cada artigo que condizem com os parâmetros de abrangimento e, posteriormente, selecionouse somente os que com potencial de auxiliar com o cerne do trabalho. Depois disto, 34 publicações seguiram para uma análise criteriosa, com o objetivo de retirar as informações centrais de cada estudo; elas foram catalogadas, conforme os pontos de relevância para este estudo de revisão. Por fim, os resultados foram debatidos.

\section{Resultados e Discussão}

\subsection{Diretrizes diagnósticas da depressão}

O termo depressão é utilizado para referir a qualquer um dos transtornos depressivos e se apresenta como sendo um fenômeno altamente complexo que envolve a presença de componentes biológicos, psicológicos e culturais (Fried\& Nesse, 2015). Constata-se que esse mal pode atingir todo o ciclo vital, o que traz consequências multifatoriais, como, o abandono de atividades realizadas rotineiramente, custo para o sistema de saúde, declínio social, sofrimento psicológico, diminuição do sentimento de prazer, falta de perspectiva para novas experiências, perda de apetite, alterações no sono e até o aumento de morbi-mortalidade quando associada ao suicídio (Santos et al., 2017). Haja vista a possibilidade abrangente de sintomatologia, deve-se ficar atento para diferenciar os diversos quadros estabelecidos pelos manuais psiquiátricos, como, o Manual Diagnostico de Transtornos Mentais (DSM-5) ou a Classificação Internacional de Doenças (CID-10).

Apesar da possibilidade de listar os sintomas dos indivíduos com depressão, apenas o enquadramento não é suficiente para determinar a presença ou ausência da doença, faz-se necessária uma entrevista clínica pautada na escuta ao sujeito e 
avaliação dos resultados obtidos por um especialista de modo a determinar a existência da comorbidade de acordo com a especificidade de cada caso (Daré \& Caponi, 2017). De acordo com a DSM-5, o transtorno depressivo maior (TDM), ou seja, a depressão, espera-se que o paciente apresente 5 ou mais dos sintomas listados no Quadro 1 por um período de duas semanas. Já em relação a CID-10, a depressão apresenta os principais sintomas: redução da possibilidade de sentir prazer, diminuição da concentração, fadiga após mínimo esforço, alterações no sono, aumento/diminuição de apetite, redução da autoestima e autoconfiança e culpabilidade; e ainda a classifica em 3 estágios, sendo eles: a leve (de 2 a 3 sintomas), o moderado (de 4 ou mais sintomas) e o grave (presença de vários sintomas de forma angustiante).

Quadro 1 - Sintomas da depressão de acordo com a DSM-5

\begin{tabular}{|l|} 
1- Humor deprimido na maior parte do dia \\
2- Diminuição de interesse ou prazer nas atividades diárias \\
3- Perda/ganho significativo de peso ou redução/aumento do apetite \\
4- Insônia ou hipersonia \\
6- Fadiga ou perda de energia \\
7- Sensação de inutilidade ou culpa \\
9- Indecisão ou perda na capacidade de concentração \\
9- Pensamentos recorrentes de morte, ideação suicida ou tentativa de suicídio
\end{tabular}

Fonte: American PsychiatricAssociation. (2014). Manual diagnóstico e estatístico de transtornos mentais: DSM-5.

Em relação ao tratamento, a variedade de possibilidades proporciona ao clínico envolver os aspectos biológicos, psicológicos e sociais. Quando é necessário fazer uso farmacológico, há uma gama de classes que podem ser usados, como, os tricíclicos (ADTs), os inibidores seletivos da recaptação da serotonina e noradrenalina (ISRSNs), os inibidores da enzima monoamina oxidase (IMAOs) e, os mais utilizados e considerados como tratamento de primeira linha, os inibidores seletivos da recaptação de serotonina (ISRSs) (Amaral, 2014). Ademais, estudos mostraram que a terapia comportamental cognitiva e terapia interpessoal, trouxeram significativas melhoras para pacientes com depressão grave (Park, Cuijpers, Van Straten\& Reynolds, 2014). Além disso, Didonéet al. (2020) afirmaram que a qualidade de vida e o suporte social são fatores protetores para os sintomas depressivos, nesse viés é de extrema importância que haja um suporte geral atrelado a psicoterapia e ao incentivo de mudanças de hábitos. 


\subsection{Diretrizes diagnósticas da ansiedade}

Os transtornos de ansiedade englobam transtornos que possuem o medo, a ansiedade e perturbações comportamentais como características em comum, segundo o DSM-5; os transtornos ainda podem ser diferenciados entre si pelos tipos de situações que provocam essas características. Bandelow e Michaelis (2015) concluíram que mesmo os transtornos de ansiedade sendo os mais comuns dentre os transtornos psiquiátricos, eles recebem menos atenção de psiquiatras clínicos, já que esses pacientes são em sua maioria tratados no ambulatório.

Uma pesquisa recente realizada por Costa, Branco, Vieira, Souza e Silva (2019) revela a agorafobia como transtorno de ansiedade com maior prevalência entre os entrevistados, seguido por transtorno de ansiedade generalizada e fobia social; são diversos os fatores de risco relacionados a esses transtornos, estando inclusos sexo, baixas condições socioeconômicas e escolaridade.

$\mathrm{O}$ transtorno de ansiedade social é caracterizado pelo medo acentuado de um comportamento impróprio em meios sociais, acompanhado na maioria das vezes por sintomas; posto isto, qualquer situação em que o indivíduo seja colocado no centro das atenções, será considerada sofrível (Chagas et al., 2010). Se tratando do transtorno de ansiedade generalizada, ele diverge do transtorno anterior ao apresentar sintomas de ansiedade e preocupação que oscilam, não necessitando de determinada circunstância, como afirma a Associação Brasileira de Psiquiatria - ABP (2008); o DSM-5 exige a persistência dos sintomas, com duração de seis meses ou mais, além do preenchimento de alguns critérios, enumerados no Quadro 2.

Quadro 2 - Critérios diagnósticos segundo o DSM-5.

\begin{tabular}{|c|c|}
\hline Agorafobia & Transtorno de Ansiedade Generalizada \\
\hline 1. Uso de transporte & $\begin{array}{l}\text { 1. Inquietação ou sensação de estar com os nervos à } \\
\text { flor da pele }\end{array}$ \\
\hline 2. Permanecer em espaços abertos & 2. Fatigabilidade \\
\hline 3. Permanecer em locais fechados & $\begin{array}{l}\text { 3. Dificuldade em concentrar-se ou sensações de } \\
\text { "branco" na mente }\end{array}$ \\
\hline $\begin{array}{l}\text { 4. Permanecer em uma fila ou ficar em meio a } \\
\text { uma multidão }\end{array}$ & 4. Irritabilidade \\
\hline \multirow[t]{2}{*}{ 5. Sair de casa sozinho } & 5. Tensão muscular \\
\hline & 6. Perturbação do sono \\
\hline
\end{tabular}

Fonte: Adaptado de "Manual Diagnóstico e Estatístico de Transtornos Mentais" - American PsychiatricAssociation (2013).

Para se obter um diagnóstico de transtorno de ansiedade generalizada em adultos, atendendo os critérios do DSM-5, as características devem estar associadas com três ou mais dos seis sintomas descritos. Balaram e Marwaha (2020) conceituam a agorafobia como a ansiedade que uma pessoa sente ao estar em um local movimentado com impossibilidade de fuga, juntamente com o medo de que ocorra um ataque de pânico. De acordo com o DSM-5, o paciente deve sentir medo ou 
ansiedade em duas ou mais das cinco situações expostas no quadro.

O tratamento é realizado através da psicoterapia e da farmacoterapia; Ravindran e Stein (2010) classificam os inibidores seletivos da recaptação da serotonina e os inibidores da recaptação da serotonina-norepinefrina como agentes de primeira linha usados na maioria dos transtornos de ansiedade, já que estão comumente relacionados com transtornos de humor. Os autores ainda ressaltam que, mesmo com um número considerável de possíveis tratamentos farmacológicos, muitos pacientes não obtêm o resultado esperado. Não é incomum a piora dos sintomas de ansiedade nos primeiros dias de tratamento, assim como a manifestação de efeitos colaterais, que acabam levando o paciente a descontinuar a medicação (Maron\&Nutt, 2017).

\subsection{Diretrizes diagnósticas da obesidade}

A obesidade é uma doença crônica de difícil controle caracterizada pelo acúmulo de gordura em tecido adiposo e que acarreta diversos prejuízos para a saúde do indivíduo (Almeida, Zanatta e Rezende, 2012). Pode ainda ser fator de risco para o desenvolvimento de comorbidades como alterações metabólicas, diabetes, doenças cardiovasculares, apneia do sono, osteoartrite e alguns tipos de cânceres (Rocha e Costa, 2012).

Quantitativamente, a obesidade é diagnosticada pela OMS (1998) tendo como base o cálculo do IMC que é expresso através da relação entre peso, em quilogramas, pela estatura ao quadrado, em metros $\left(\mathrm{kg} / \mathrm{m}^{2}\right)$. Dessa forma, são considerados obesos os indivíduos que apresentem IMC maior ou igual a $30 \mathrm{~kg} / \mathrm{m}^{2}$. Apovian (2016) explica que medidas de adiposidade central, como aumento da circunferência abdominal, são importantes na determinação de patologias advindas da obesidade, justificando que deve-se ainda estudar como incorporar outros diagnósticos além do IMC na clínica. O Quadro 3 expressa algumas relações entre valores de IMC com suas respectivas classificações de peso e risco a comorbidades.

Quadro 3 - Classificação para adultos de acordo com IMC.

\begin{tabular}{|l|l|l|}
\hline Classificação & IMC & Risco de Comorbidade \\
\hline Abaixo do peso & & $\begin{array}{l}\text { Baixo (porém aumentado para outras } \\
\text { comorbidades clinicas }\end{array}$ \\
\hline Peso normal & $18.5 \mathrm{~kg} / \mathrm{m}^{2}$ & Médio \\
\hline Sobrepeso & $25.0-29.9 \mathrm{~kg} / \mathrm{m}^{2}$ & Aumentado \\
\hline Obesidade grau 1 & $30.0-34.9 \mathrm{~kg} / \mathrm{m}^{2}$ & Moderado \\
\hline Obesidade grau 2 & $35.0-39.9 \mathrm{~kg} / \mathrm{m}^{2}$ & Severo \\
\hline Obesidade grau 3 & $\geq 40.0 \mathrm{~kg} / \mathrm{m}^{2}$ & Muito severo \\
\hline
\end{tabular}

Fonte: Adaptado de "Obesity: preventing and managing the global epidemic." de Wolrd Health Organization (2000).

No que tange à etiologia da obesidade, Apovian (2016) ressalta que a causa exata da obesidade ainda é pouco conhecida, embora haja relação multifatorial complexa para tal. Podem estar envolvidos fatores culturais, biológicos, psicossociais, comportamentais, genéticos, socioeconômicos. Algumas comorbidades e seus tratamentos também podem ter influência no aparecimento da obesidade. Atualmente, o tratamento e a prevenção têm se concentrado no aspecto psicológico e social da doença, sendo os melhores resultados não invasivos associados à mudança nos hábitos alimentar e comportamental e a maior efetividade relacionada à cirurgia bariátrica, com essa última apresentando baixo risco de mortalidade (Almeida, Zanatta \& Rezende, 2012).

Wanderley e Ferreira (2010) descrevem que a ascensão da obesidade no mundo pode ser, em parte, explicada pelo fenômeno da transição nutricional típica da modernidade, no qual a população mundial como um todo tem migrado de doenças 
nutricionais características do subdesenvolvimento, como a desnutrição, e passado a apresentar outras vinculadas à modernidade, como a obesidade. Isso se deve à dieta moderna ser caracterizada por uma alta ingestão de gorduras em detrimento de carboidratos e da adoção de um estilo de vida mais sedentário em detrimento a um mais ativo e com maior gasto energético. Tal evento acarreta um balanço energético diário positivo, que é a causa primária do ganho de peso e advento da obesidade.

Ademais, o estudo mostrou ainda que a qualidade da alimentação está diretamente ligada ao poderio econômico das famílias e da possibilidade de gastos com alimentação, que reflete na maior ingestão de alimentos industrializados e fastfoodspor indivíduos com baixo poder aquisitivo. Esse fato foi comprovado pela pesquisa de Guimarães, et al. (2012), que mostrou que a relação entre classe social e excesso de peso é maior conforme diminui-se o poder aquisitivo do indivíduo, além de encontrar maior quantidade de casos de obesidade entre escolares da rede pública em detrimento dos da rede privada de ensino.

A genética também é um importante fator para o surgimento da obesidade. Wanderley e Ferreira (2010) discorrem que o peso corporal é controlado por relações entre uma serie de fatores tais quais hormônios, neuropeptídios, vias aferentes e eferentes, evidenciando que mutações nos genes responsáveis por tais componentes têm sido associadas à obesidade. A revisão de Apovian (2016) confirma esse resultado, afirmando que a genética parece determinar quem será obeso, com o ambiente influenciando no grau da obesidade.

Ainda, a obesidade pode estar relacionada a fisiopatologias como desordens no eixo hormonal. Nesse sentido, Wanderley e Ferreira (2010) caracterizam a leptina como um importante hormônio marcador da quantidade de tecido adiposo, sendo responsável por reduzir a fome através da inibição da formação do neuropeptídeo Y (NPY) e promover o aumento na expressão de neuropeptídeos anorexígenos como o hormônio liberador de corticotropina (CRH). A grelina também é importante hormônio responsável pela regulação do peso corporal, sendo capaz de estimular a ingestão de alimentos quando liberado. São ainda importantes para o controle de peso hormônios como a Adiponectina (aumenta sensibilidade à insulina e promove oxidação de ácidos graxos), Colecistoquinina (suspensão da fome), Peptídeo Tirosina-Tirosina (efeitos anorexígenos), Oxintomodulina (suprime a fome e reduz ingestão de alimentos) e Peptídeo semelhante ao glucagon (promove liberação de insulina e inibição da liberação de glucagon, com seus efeitos não glicêmicos associados à perda de peso) (Apovian, 2016). Desse modo, alteração nos eixos de qualquer um desses hormônios pode acarretar ganho de peso e levar à obesidade.

\subsection{Tratamento multidisciplinar da comorbidade depressão, ansiedade e obesidade}

Em relação à comorbidade entre depressão, ansiedade e obesidade, a literatura apresenta muitas divergências no que tange à relação entre as três patologias, apesar de parecer haver certo consenso de que a comorbidade é maior na população obesa do sexo feminino. Almeida, Zanatta e Rezende (2012) em estudo feito com indivíduos obesos avaliaram a autoimagem corporal, os níveis de depressão e os de ansiedade, em fases pré-cirurgia bariátrica, 6 meses após a cirurgia e 12 meses após a cirurgia. Como resultado, encontraram que os níveis de ansiedade permaneceram mínimos em todas as etapas do processo, apesar de apontar certa redução em tais níveis entre o pré e pós-operatório. Com relação à depressão, os níveis em préoperatório eram leves e mostraram-se mínimos nas duas fases de pós-operatório, fato por eles atribuído à redução na insatisfação corporal apresentada por todos os indivíduos. Dessa forma, concluíram que a ansiedade e a depressão são aspectos emocionais significativos no quadro de obesidade dos sujeitos.

Outro estudo, elaborado por Rocha e Costa (2012), no entanto, apresentou resultados de certa forma divergentes. Neste, a maioria dos sujeitos obesos entrevistados não apresentou valores com significado clínico relevante para ansiedade, apesar de uma porção significativa apresentar valores de resultado compatível com diagnóstico de ansiedade moderada (21\% 
ou 21 indivíduos). A amostra também não apresentou valores estatisticamente significativos aos níveis de sintomatologia depressiva, uma vez que os valores encontrados estavam dentro do esperado para a população normal. Ainda, foram encontrados resultados de autoconceito dos indivíduos compatíveis com os da população em geral, levando os autores a concluir que os níveis de ansiedade e depressão são tanto menores quanto maior o autoconceito nos obesos mórbidos. Assim, a pesquisa acabou por concluir que não há correlação estatística significativa entre IMC, depressão e ansiedade.

Ademais, Wanderley e Ferreira (2010) defendem que a postura atual é a de que a população obesa não apresenta maiores indicies de psicopatologias se comparada à população não obesa, sendo estes sintomas restritos a grupos específicos de indivíduos, em que a obesidade seria visto como consequência desses, não como causa. Dessa forma, exemplificam dois transtornos alimentares de ordem comportamental que são mais frequentemente vistos em sujeitos obesos, sendo eles o transtorno da compulsão alimentar periódica (TCAP), que leva o individuo a ingerir grandes quantidades de alimento em curtos períodos de tempo; e a síndrome do comer noturno (SCN), caracterizada por maior ingestão de alimentos no período da noite, anorexia vespertina e insônia. Ambos são associados à obesidade, baixa autoestima e depressão. Pereira e Brandão (2014) confirmam a influência dos transtornos de compulsão alimentar no surgimento da obesidade e reafirmam sua relação com sintomas depressivos e de redução de autoestima, mas explicam que não existe relação direta entre tais transtornos e IMC, levando a crer que existem outros fatores que medeiam essa associação.

Há também forte relação entre o uso de psicofármacos para tratamento de transtornos de humor e de ansiedade e o ganho de peso. Apovian (2016) discorreu que o uso de antipsicóticos usados no tratamento de TDM está associado a ganho de peso, diabetes e distúrbios lipídicos. Ainda, seu uso no tratamento em longo prazo pode levar a acúmulos de lipídeos, triglicerídeos e glicose no sangue, acarretando ganho de peso. Dessa forma, seu uso deve ser acompanhado de aferição de altura, peso, IMC e circunferência abdominal pelo médico responsável, com troca da classe de antipsicóticos utilizada caso o ganho de peso ultrapasse $5 \%$ do peso basal do sujeito.

Smits, et al. (2010), usando uma amostra significativa de homens e mulheres canadenses maiores de 15 anos, demonstrou que a correlação entre obesidade e transtornos de humor é amplamente influenciada pelo uso de fármacos, enquanto que a relação entre ansiedade e obesidade é parcialmente afetada por tal uso. Os efeitos da relação entre medicamento e obesidade foram específicos para antidepressivos e antipsicóticos, tendo esses dois emergindo como preditores significativos de tal patologia, apresentando valores de predisposição à obesidade (após 12 meses de uso) de mais de um e meio para antidepressivos e de mais de dois para antipsicóticos, em relação a indivíduos que não fazem uso destes. $\mathrm{O}$ estudo também afirma que a reversão da direcionalidade nessa situação é improvável, isto é, que o surgimento da obesidade levaria à necessidade de uso desses fármacos. Por fim, a pesquisa pressupõe que o ideal para redução no ganho de peso em tratamentos de transtornos mentais seria a adoção de terapias não medicamentosas. Contudo, dada a impossibilidade da adoção de tratamentos não farmacológicos para transtornos mentais em boa parte dos casos, sugere-se que seja feita uma intervenção medicamentosa juntamente a estratégias para controle do ganho de peso.

\section{Conclusão}

A obesidade é um problema de saúde multifatorial e, quando associado a comorbidades, acarreta maiores complicações tanto nos aspectos terapêuticos como evolutivos. Independente de sua gravidade é primordial a observância dos seus fatores etiológicos, desencadeantes, predisponentes e perpetuadores. Encontram-se entre os seus fatores de risco, ainda, a associação com distúrbios emocionais, como a ansiedade e a depressão, os quais muitas vezes aumentam a necessidade da ingestão de alimentos calóricos ou desregulam o ritmo alimentar e metabólico, trazendo complicações ao tratamento e comprometendo o prognóstico do quadro clínico. Com relação à comorbidade obesidade, depressão e ansiedade, deve-se 
atentar para o fato de que tal conjuntura apresenta relação de causalidade ainda controversa na literatura, embora as linhas de pesquisa mais atuais apontem para a não existência de uma correlação direta entre obesidade e desenvolvimento de transtornos mentais, mas sim a presença destes levando a uma maior probabilidade de desenvolvimento de quadros de obesidade. Portanto, ao lidar com quadros de obesidade comórbida com ansiedade e depressão, deve-se atentar aos medicamentos utilizados para o tratamento destas, bem como as consequências emocionais e psicológicas causadas por tais patologias, garantindo que suas influências sejam mínimas no tratamento da obesidade. Futuras pesquisas poderão direcionar-se para a busca de uma solução medicamentosa para os transtornos de ansiedade e depressão que não cause alterações no peso do indivíduo, ou ainda para a busca de outras não medicamentosas, de modo a garantir melhor tratamento e maior possibilidade de melhora no quadro de indivíduos acometidos por essas patologias.

\section{Referências}

Almeida, S., Zanatta, D., \& Rezende, F. (2012). Imagem corporal, ansiedade e depressão em pacientes obesos submetidos à cirurgia bariátrica. Estudos de Psicologia (Natal), 17(1),153-160. 10.1590/s1413-294x2012000100019

Amaral, A. D. (2014). Comparação entre SNRI e SSRI na indução da remissão da perturbação depressiva major: uma revisão baseada na evidência. Revista Portuguesa de Medicina Geral e Familiar, 30(3), 174-180.

American PsychiatricAssociation. (2014). Manual diagnóstico e estatístico de transtornos mentais: DSM-5 (5a ed.), Artmed.

Apovian, C. M. (2016). Obesity: Definition, comorbidities, causes, and burden. Am. J. Manag. Care, 22, S176-S185.

Associação Brasileira de Psiquiatria. Transtornos de ansiedade: Diagnóstico e Tratamento (2008) https://diretrizes.amb.org.br/_BibliotecaAntiga/transtornosde-ansiedade-diagnostico-e-tratamento.pdf.

Balaram, K., Marwaha, R., \& Agoraphobia. Treasure Island: Statpearls Publishing (2020). https://www.ncbi.nlm.nih.gov/books/NBK554387/.

Bandelow, B., \& Michaelis, S. (2015) Epidemiology of anxiety disorders in the 21st century. Dialogues ClinNeurosci, 17(3): 327-335.

Chagas, M. H. N., Nardi, A. E., Manfro, G. G., Hetem, L. A. B., Andrada, N. C., Levitan, M. N., Salum, G. A., Isolan, L., Ferrari, M. C. F., \& Crippa, J. A. S. (2010). Diretrizes da Associação Médica Brasileira para o diagnóstico e diagnóstico diferencial do transtorno de ansiedade social. Brazilian Journal of Psychiatry, 32(4), 444-452. Epub October 15, 2010. https://dx.doi.org/10.1590/S1516-44462010005000029

CID-10. Classificação de Transtornos Mentais e de Comportamento da CID-10: Descrições clínicas e diretrizes diagnósticas. 1993: Artmed.

Costa, C. O. da., Branco, J. C., Vieira, I. S., Souza, L. D. de M., \& Silva, R. A. da. (2019). Prevalência de ansiedade e fatores associados em adultos. Jornal Brasileiro de Psiquiatria, 68(2), 92-100. https://doi.org/10.1590/0047-2085000000232

Daré, P. K., \& Caponi, S. N. (2017). Cuidado ao indivíduo com depressão na atenção primária em saúde. ECOS-Estudos Contemporâneos Da Subjetividade, 7(1). https://www.periodi-coshumanas.uff.br/ecos/article/view/1858.

Didoné, L. S., Jesus, I. T. M. de., Santos-Orlandi, A. A., Pavarini, S. C. I., Orlandi, F. de S., Costa-Guarisco, L. P., Gratão, A. C. M., Gramany-Say, K., Cominetti, M. R., Gomes, G. A. de O., \& Zazzetta, M. S. (2020). Factors associated with depressive symptoms in older adults in context of social vulnerability. Revista Brasileira de Enfermagem, 73(Suppl. 1), e20190107.https://doi.org/10.1590/0034-7167-2019-0107

Frontzek, L. G. M., Bernardes, L. R., \& Modena, C. M. (2017). Obesidade infantil: compreender para melhor intervir. Revista da Abordagem Gestáltica, 23(2), 167-174.

Garcia, G., Pompeo, D., Eid, L., Cesarino, C., Pinto, M., \& Gonçalves, L. (2018). Relationship between anxiety, depressive symptoms and compulsive overeating disorder in patients with cardiovascular diseases. Revista Latino-Americana De Enfermagem, 26(0). 10.1590/1518-8345.2567.3040

Guimarães, A., Feijó, I., Soares, A., Fernandes, S., Machado, Z., \& Parcias, S. (2012). Excesso de peso e obesidade em escolares: associação com fatores biopsicológicos, socioeconômicos e comportamentais. Arquivos Brasileiros de Endocrinologia \& Metabologia, 56(2),142-148. 10.1590/s000427302012000200008

Maron, E., \& Nutt D. (2017). Biological markers of generalized anxiety disorder. Dialogues ClinNeurosci, 19(2), 147-158.

Park, M., Cuijpers, P., van Straten, A., \& Reynolds, C. F. (2014). The effects of psychotherapy for adult depression on social support: A meta-analysis. Cognitive therapy and research, 38(6), 600-611. https://doi.org/10.1007/s10608-014-9630-z

Pereira, C., Brandão, I. (2014). Uma perspetiva da psicopatologia da obesidade. Arq Med [Internet], 28(5), 152-159.

Ravindran, L. N., \& Stein, M. B. (2010). The pharmacologic treatment of anxiety disorders: a review of progress. The Journal of clinical psychiatry, 71(7), 839-854. https://doi.org/10.4088/JCP.10r06218blu

Rocha C, \& Costa E. (2012). Aspectos psicológicos na obesidade mórbida: Avaliação dos níveis de ansiedade, depressão e do auto-conceito em obesos que vão ser submetidos à cirurgia bariátrica. Análise Psicológica, 30(4):451-466.10.14417/ap.604 
Research, Society and Development, v. 10, n. 1, e16210111489, 2021

(CC BY 4.0) | ISSN 2525-3409 | DOI: http://dx.doi.org/10.33448/rsd-v10i1.11489

Santos, H. G. B., Marcon, S. R., Espinosa, M. M., Baptista, M. B., \& Paulo, P. M. C. (2017). Fatores associados à presença de ideação suicida entre universitários. Revista Latino-Americana de Enfermagem, 25(2878), p.1-8. 10.1590/1518-8345.1592.2878

Smits, J., Rosenfield, D., Mather, A., Tart, C., Henriksen, C. \&, Sareen, J. (2010). Psychotropic medication use mediates the relationship between mood and anxiety disorders and obesity: Findings from a nationally representative sample. J Psychiatr Res, 44(15):1010-1016. 10.1016/j.jpsychires.2010.04.007

Wanderley, E., \& Ferreira, V. (2010). Obesidade: uma perspectiva plural. Ciência \& Saúde Coletiva, 15(1):185-194. 10.1590/s1413-81232010000100024

Whittemore, R., \& Knafl, K. (2005). The integrative review: updated methodology. J AdvNurs, 52(5):546-553. 10.1111/j.1365-2648.2005.03621.x

WHO (2020). Obesity: preventing and managing the global epidemic. Report of a WHO Consultation. WHO Technical Report Series 894 . Geneva: World Health Organization. 